\section{... et ça recommence!}

Merci cher Président, félicitations cher Jacques! Excellent édito! Je suis tout à fait d'accord avec toi, et cette politique me plaît [1]. Nos politiciens sont complètement empêtrés dans un carcan qui les empêche de trouver une solution. Et pourquoi ne serions-nous (la FMH) pas à l'origine d'une nouvelle vision?

Je pense que nous allons de plus en plus vers une assurance unique. Mais celle-ci devra se faire avec nous! Donc il est crucial que nous commencions à nous y intéresser sérieusement dès maintenant, en listant nos exigences, pour ne pas nous faire berner par l'OFAS une fois de plus.

Dans un premier temps, il me paraîtrait intéressant de soutenir la proposition du vaudois Michel Surbeck qui demande un pool commun des réserves des caisses-maladie (ce qui avait déjà été demandé par la SMSR il y a quelques années). Un autre sujet qui pourrait être débattu serait celui des génériques. Pour ma part, j'estime que cet artifice des industries n'est pas la solution. Les génériques permettent des bénéfices sans doute gigantesques (certainement supérieurs à ceux des originaux, preuve donc qu'ils sont trop chers!), sinon comment expliquer la prolifération de ces médicaments, dont la conséquence principale est une anarchie totale chez les pharmaciens. Pourquoi ne pas contraindre simplement, par exemple l'industrie à baisser les prix des originaux de moitié au terme de la protection des 10 ans? Je suis persuadé que cette simplification serait largement plus efficace que ce système boiteux et qui ne déclenche visiblement pas l'enthousiasme de la population ni des médecins.

Je reste convaincu qu'il existe dans le corps médical des confrères pleins d'idées qui ne demanderaient qu'à s'exprimer sur ces sujets, preuve en est le courrier qu'a déclenché le problème des vaccinations!

$$
\text { Jean-Pierre Grillet, Genève }
$$

1 de Haller J. ... et ça recommence! Bull Méd Suisses 2006;86(39):2213.

\section{Jahr für Jahr das gleiche Spiel}

Unser FMH-Präsident spricht sich in seinem Editorial [1] gegen neoliberale «Kopfpauschalen» aus und offeriert als Stichworte für die Finanzierung einkommensabhängige Prämien oder die Mehrwertsteuer.

Gerne erinnere ich ihn an den 4. Dezember 1994: Das Schweizer Volk hat dem neuen KVG mit knappen 51,8\% der Stimmenden zugestimmt und es wollte so die Solidarität zwischen Jungen und Alten und zwischen Gesunden und Kranken verankern sowie die Kostensteigerung dämpfen. Dies ist dem KVG doch recht gut gelungen! Am selben Datum hat sich das Schweizer Volk aber mit überwältigenden 76,6\% der Stimmenden gegen die Initiative «für eine gesunde Krankenversicherung» ausgesprochen. Ziel der Initiative war unter anderem der Beitrag der Versicherten nach Massgabe der wirtschaftlichen Leistungsfähigkeit.

Unsere Kosten (Preis pro Dienstleistung oder Ware) sind seit Jahren rückläufig. Das Problem sind nicht die Kosten, sondern die Mengenausweitung, und dagegen, mit Verlaub, helfen weder einkommensabhängige Prämien noch die Erhöhung der Mehrwertsteuer!

Dr. med. Xaver Troxler-Mattmann, Luzern

1 de Haller J. Jahr für Jahr das gleiche Spiel. Schweiz Ärztezeitung 2005;86(39):2214. 
Der Herr hat's gegeben, der Herr hat's genommen ... oder von gottgewollten und nicht gewollten Preisen im Schweizer Gesundheitswesen

Anno 350 ante Christum streiten sich zwei auf dem Marktplatz. Der eine will dem anderen einen Gaul verkaufen, doch der Preis, den er vor einigen Monaten bezahlt hat, ist um mehr als das Eineinhalbfache gestiegen. Doch er braucht den Gaul, um seinen Karren zu ziehen. Und den muss er ziehen, um auf dem Markt seine Ware an den Mann und an die Frau zu bringen. Widerwillig willigt er in den Handel ein und bezahlt. Eine Woche später ist der Gaul zu verkaufen, der Herr hat den Mann zu sich gerufen, und keine Medizin jener Welt konnte ihm helfen.

Im Jahre 2005 ist der Benzinpreis um gut 60\% gestiegen, und männiglich zahlt mit mehr oder weniger geballter Faust im Sack. Herr Strahm hat sich nicht vernehmen lassen. Der Markt ist schliesslich gottgewollt, und er spielt. Im September des gleichen Jahres kündigt das Konkordat der Schweizer Krankenversicherer wiederum satte Prämienaufschläge für das kommende Jahr an: Mindestens 5\% im Durchschnitt sollen es sein. Und man ist sich einig, so könne es nicht weitergehen. Das sei nicht gottgewollt, der Markt spiele nicht, eine Verschwörung ist im Gang. Der Aufschrei hallt durch die Wandelhallen des Bundeshauses und durch die Arena von SF DRS. Viele Massnahmen wurden ergriffen und als Erfolg bezeichnet, so die Streichung von Spitälern von der Spitalliste, der Zuwachs des Generikaanteils am Pharmamarkt, die Vereinbarung, inskünftig die Medikamente in der Schweiz günstiger anzubieten, der Zulassungsstopp für neue Arztpraxen, um nur einige zu nennen. Alles hat nichts gefruchtet. Alle wüssten eigentlich, wie wirksam Ausgaben für das Gesundheitswesen einzusparen wären. Man müsste so ehrlich sein und den Menschen in diesem Land sagen, dass es wesentlich billiger würde, würde der Herr wie anno 350 ante Christum wieder Leben schenken und nehmen. Sicher, Herr Antinori hat es ja bewiesen, heute kann auch die 60jährige Grossmutter nochmals Mutter sein, und die Reproduktionsmedizin hat sich kräftig in Gottes Geschäft eingemischt. Auch das mit der Jungfrau Maria, die auf wundersame Weise zu ihrem Kinde kam, eben von Gott gewollt, ist so ziemlich in eine wissenschaftliche Schieflage geraten. Und gegen das Ende eines Lebenskreises kann die Herzinsuffizienz und das metastasierende Karzinom um Jahre länger als noch vor ein, zwei Dezennien überlebt werden, doch das hat seinen hohen Preis.

Vielleicht könnte man sich auch fragen, wofür wir wieviel auszugeben bereit sind. Beim Benzinund Heizölpreis ist die Schmerzgrenze offenbar noch nicht erreicht. Aber wir haben ja gelernt: Erdöl hat uns der Herr gegeben, so viel, wie ihm genehm ist, und wir müssen die Kräfte wirken lassen, die von Angebot und Nachfrage. Der medizinische Fortschritt hingegen ist eine häretische Einmischung der Menschen in die Natur. Dass dabei die Preise aus den Ufern laufen, will uns lehren: Der Herr hat's gegeben, aber wir stören seine Kreise beim Nehmen. Und weil eben immer die letzten Jahre des Lebens die teuersten werden, verbilligt der medizinische Fortschritt die Gesundheitskosten nicht, sondern er verteuert sie um die Jahre, die wir länger leben. Mit einem freundlichen Gruss an die Gesundheitspolitiker.

Dr. med. D. Schlossberg, Zürich

\section{Une caisse-maladie unique}

Un très grand merci au Dr Philippe Wacker pour ses réflexions sur une refonte de notre système de santé [1].

J'ai été réfractaire pendant toute ma carrière à l'idée d'une caisse-maladie unique. De guerre lasse pourtant et n'espérant plus rien à ce sujet de la part des parlementaires et de santésuisse, j'en arrive à me persuader de la nécessité d'une telle institution. Intelligemment conçue, elle serait, semble-t-il, la seule possibilité de déparasiter le domaine de la santé publique de ses corps étrangers, laissant enfin la parole et les choix à ses deux seuls partenaires réels, à savoir les assurés et les soignants, le rôle de l'Etat consistant à veiller à la bonne application des règles de fonctionnement que se donnerait cette nouvelle entité.

N'en restons pas à l'idée que tout cela est trop beau pour être vrai!

Dr J. P. Bruggimann, Soral

1 Wacker P. A propos des hausses annoncées de cotisations par les caisses-maladie. Bull Méd Suisses 2006;85(41):2319. 